\title{
Erratum to: On Rank Driven Dynamical Systems
}

\author{
J. J. P. Veerman ${ }^{1}$ - F. J. Prieto ${ }^{2}$
}

Published online: 30 September 2015

CC Springer Science+Business Media New York 2015

\section{Erratum to: J Stat Phys (2014) 156:455-472 DOI: 10.1007/s10955-014-1012-0}

In the article "On Rank Driven Dynamical Systems", by J. J. P. Veerman and F. J. Prieto, Journal of Statistical Physics, 156:455-472 (2014), the following two errata were detected:

- In Theorem 2, where it states

"Furthermore $\Gamma_{\text {exo }}^{*}$ is strictly increasing and $C^{\infty}$ on $(0,1)$ while $R_{\text {endo }}^{*}=\Gamma_{\text {endo }}^{*}\left(R_{0}\right)$ is the cdf corresponding to a weighted sum of finitely many delta distributions."

it should instead say

"Furthermore $\Gamma_{\text {exo }}^{*}$ is strictly increasing and $C^{\infty}$ on $(0,1)$ while $R_{\text {endo }}^{*}=\Gamma_{\text {endo }}^{*}\left(R_{0}\right)$ is the cdf corresponding to a weighted sum of finitely many step distributions."

- In Eq. (8) there is a condition missing. Instead of

$$
\alpha_{i}=\frac{q-p}{n-k(n)}+o(1 / n), \quad \tilde{p} \equiv \sum_{i=1}^{k(n)} \alpha_{i}, \quad \tilde{\alpha}=\frac{q-p}{n-k(n)}
$$

the equation should read

$$
\alpha_{i}=\frac{q-p}{n-k(n)}+o(1 / n), i>k(n), \quad \tilde{p} \equiv \sum_{i=1}^{k(n)} \alpha_{i}, \quad \tilde{\alpha}=\frac{q-p}{n-k(n)} .
$$

The online version of the original article can be found under doi:10.1007/s10955-014-1012-0.

$凶 \quad$ F. J. Prieto

franciscojavier.prieto@uc3m.es

J. J. P. Veerman

veerman@pdx.edu

1 Fariborz Maseeh Department of Mathematics and Statistics, Portland State University,

Portland, OR, USA

2 Department of Statistics, Univ Carlos III, Madrid, Spain 